\title{
Estudio geotécnico con fines de cimentación para tres asociaciones de vivienda en Tacna
}

\author{
Geotechnical study for foundation purposes for three housing associations in \\ Tacna
}

\author{
${ }^{1}$ Brandon Huanca Cutipa \\ ${ }^{2}$ Ruth Flores Terrazas \\ ${ }^{3}$ Wilber Mendoza Ramírez
}

\section{RESUMEN}

La presente investigación cuantitativa tuvo como objetivo principal plantear una adecuada alternativa de cimentación a partir de la realización de estudios geotécnicos mediante los ensayos de laboratorio y métodos geofísicos: refracción sísmica y MASW en las asociaciones de vivienda Los Damnificados del 23 de Junio B, Corazón de María y El Manantial, ubicados en el Distrito Coronel Gregorio Albarracín Lanchipa de la ciudad de Tacna. Con los ensayos de refracción sísmica se conoció la estratigrafía del terreno y su ripabilidad, con el ensayo de MASW se encontraron las propiedades dinámicas del suelo y su clasificación según NTE E.030, mientras que los ensayos de laboratorio determinan las propiedades físicas, químicas y mecánicas del suelo. Del ensayo de MASW se obtuvo que el tipo de suelo es $\mathrm{S} 1$ y corresponde a un suelo muy rígido, y de la exploración por calicatas se clasificó como grava pobremente graduada (GP). De los resultados se obtuvo un $\mathrm{Vs}_{30}$ (promedio) de $652.86 \mathrm{~m} / \mathrm{s}$, un Ts (promedio) de 0.18 y una capacidad de carga admisible con promedio de $2.1 \mathrm{~kg} / \mathrm{cm}^{2}$ por falla local. De este modo, se realizó una microzonificación de la zona con las velocidades de ondas de corte promedio $\mathrm{Vs}_{30}$, propiedades dinámicas del suelo y capacidad portante. Finalmente, conociendo las propiedades del suelo se diseñó una zapata aislada cuadrada de $1 \mathrm{~m}$ de ancho cimentada a una profundidad de $1.5 \mathrm{~m}$ para las tres asociaciones de vivienda.

Palabras claves: Cimentación, estudios Geotécnicos, métodos geofísicos, MASW, refracción sísmica, ripabilidad, suelos.

\section{ABSTRACT}

The main objective of this quantitative research is to propose an adequate alternative foundation based on geotechnical studies through laboratory tests and geophysical methods such as seismic refraction and MASW, which were applied in the housing associations of Los Damnificados del 23 de Junio - B, Corazón de María and El Manantial. All these three housing associations are in the district of Coronel Gregorio Albarracín Lanchipa in the city of Tacna, Peru. The stratigraphy of the terrain and its rippability were known with the help of the seismic refraction tests. With the MASW test, the dynamic properties of the soil and its classification according to NTE E.030 were found, while the laboratory tests determined the physical, chemical and soil mechanics. It was obtained from the MASW test, that the soil type is S1 which corresponds to a very rigid soil, and from the exploration of pits, it is classified as poorly graded gravel (GP). From these results, we obtained a $\mathrm{V}_{\mathrm{s} 30}$ (average) of $652.86 \mathrm{~m} / \mathrm{s}$, a Ts (average) of 0.18 and an average admissible load capacity of $2.1 \mathrm{~kg} / \mathrm{cm}^{2}$ for local failure. In this way, a microzoning of the area was carried out with the average cut wave velocities $\mathrm{V}_{\mathrm{s} 30}$, dynamic soil properties and bearing capacity. Finally, after obtaining the properties of the soil, a square shaped isolated footing of $1 \mathrm{~m}$ wide was designed and cemented to a depth of $1.5 \mathrm{~m}$ for the three housing associations.

Keywords: Foundations, geotechnical studies, geophysical methods, MASW, rippability Perú, seismic refraction, soils.

\footnotetext{
${ }^{1}$ Universidad Privada de Tacna. Tacna- Perú. E-mail: brandon10_700@hotmail.com

${ }^{2}$ Universidad Privada de Tacna. Tacna- Perú. E-mail: siiomaraaa@ $\bar{\varnothing}$ gmail.com

${ }^{3}$ Universidad Privada de Tacna. Tacna- Perú. E-mail: wimeral1601@hotmail.com
} 


\section{INTRODUCCIÓN}

La ciudad de Tacna es considerada una zona de silencio sísmico o laguna sísmica. Se espera un sismo de mayor intensidad al ocurrido el 23 de junio del 2001, que registró una intensidad de 6.9 en la escala de Richter. La población no está preparada para este fenómeno y mucho menos las infraestructuras de las viviendas, debido a que la mayoría de ellas son producto de construcciones informales, las cuales no cuentan con estudios de suelos, planos y la intervención de un profesional durante su proceso constructivo.

De este modo, después del terremoto del 2001, el Instituto Nacional de Defensa Civil (INDECI, 2004) desarrolló el mapa de peligros sísmicos en la ciudad de Tacna, el cual identificó los diferentes tipos de suelos que se ubican en la ciudad, clasificando los suelos del distrito Coronel Gregorio Albarracín Lanchipa entre gravas bien graduadas y pobremente graduadas. Debido al incremento de la población, se están poblando zonas que no fueron evaluadas detalladamente, por ejemplo, el sector denominado Viñani, ubicado en el distrito Coronel Gregorio Albarracín Lanchipa.

La presente investigación tiene como objetivo principal plantear una adecuada alternativa de cimentación a través de un estudio geotécnico, el cual beneficiará a las asociaciones de vivienda $L o s$ Damnificados del 23 de Junio B, Corazón de María y El Manantial. Los estudios geotécnicos fueron realizados mediante la exploración de calicatas, realización de ensayos básicos y especiales del suelo, y métodos geofísicos, obteniendo así las características del suelo, lo cual nos permite diseñar una adecuada cimentación.

Otros estudios se han basado en el estudio geofísico. Según Rosales (2001), los resultados explican el comportamiento dinámico del suelo y sus efectos sobre edificaciones. De igual forma, Huanacuni y Llanqui (2014) demuestran la clasificación de perfiles por medio de la velocidad de onda promedio de corte a $30 \mathrm{~m}$ y sus propiedades elásticas por la relación de velocidad de ondas Vpy Vs.

\section{MATERIALYMÉTODOS}

\section{Población o muestra de estudio}

Se consideró como población o muestra de estudio únicamente a las asociaciones de vivienda Los Damnificados del 23 de Junio B, Corazón de María y El Manantial ubicados en el distrito Coronel Gregorio Albarracín Lanchipa, Tacna.

\section{Técnicas e instrumentos para la recolección de} datos

Vara (2012) expresa que todos los instrumentos que se emplean para la recolección de datos científicos deben ser fiables y válidos. En cuanto a la recolección de datos se realizaron revisiones de informes del INDECI (Instituto Nacional de Defensa Civil), publicaciones del IGP (Instituto Geofísico del Perú), publicaciones del CISMID (Centro Peruano Japonés de Investigaciones Sísmicas y Mitigación de Desastres) e investigaciones de tesis similares que sirvieron como base para la realización de la siguiente investigación. De esta manera se realizó la visita a la zona de estudio. En la Figura 1 se observa el plano de ubicación del sector evaluado.

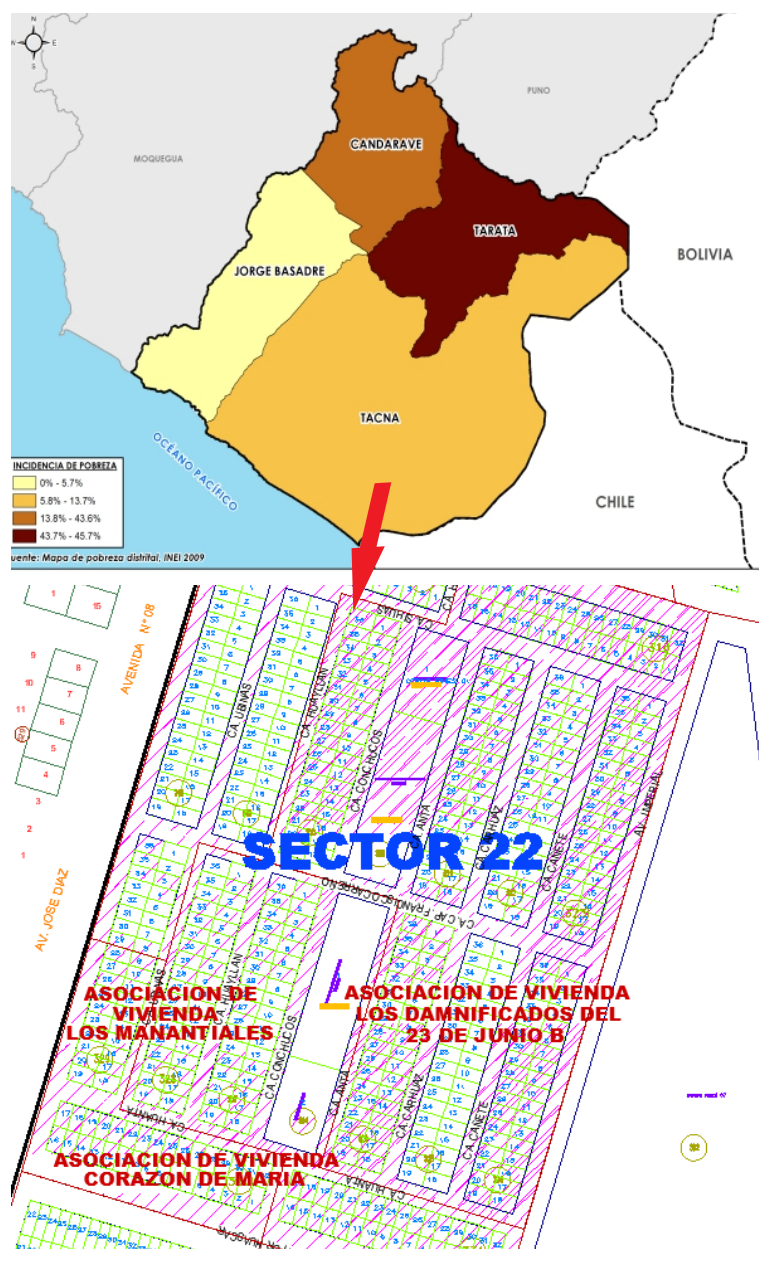

Figura 1. Plano de ubicación del sector evaluado.

De esta manera, se realizaron estudios geotécnicos mediante los ensayos de campo directos e indirectos: 


\section{Ensayos geotécnicos de campo indirectos}

En cuanto a los ensayos indirectos, se realizó la exploración geofísica mediante los métodos de Refracción Sísmica y Medición de Ondas de Corte (MASW) como se observa en la Figura 2.

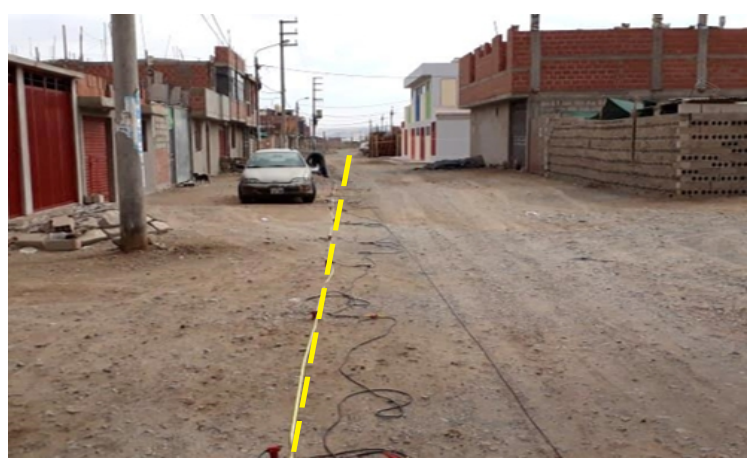

Figura 2. Línea de ensayo para el método de refracción sísmica y MASW.

El ensayo de refracción sísmica es un método de exploración geofísica que permite determinar la estratigrafía del subsuelo en forma indirecta, basándose en el cambio de las propiedades dinámicas de los materiales que lo conforman (Rivera, Piedra y Paripanca, 2016) Por otro lado, el Análisis Multicanal de Ondas Superficiales (MASW) es un método no destructivo que se usa para evaluar la velocidad de onda de corte (Vs), vinculado al mismo módulo de corte $(\mathrm{G})$. Este método analiza las propiedades de dispersión de los modos fundamentales de las ondas Rayleigh, las cuales se propagan a lo largo de la superficie desde el punto de impacto a los receptores (Park, Miller \& Xia, 1999). Esto indica que los perfiles sísmicos del terreno se precisan del método de inversión por las curvas de dispersión, por medio de las ondas Rayleight que se propagan en un medio de espacio homogéneo, consiguiendo una velocidad de fase, la cual varía entre un 90 \% y 95 $\%$ del valor de la velocidad de onda de corte (Vs) (Hayashi, 2008).

En el método de Refracción Sísmica se realizó un análisis total de $540 \mathrm{~m}$ lineales, ubicados en 7 líneas sísmicas; y se realizaron 6 ensayos de MASW, que fueron distribuidas adecuadamente en toda la zona de estudio para definir así la estratigrafía y el tipo de suelo del terreno.

Por otra parte, se realizó el levantamiento topográfico mediante fotogrametría. En la Figura 3 se observa el drone phantom 4pro v.2.0 utilizado para el levantamiento topográfico.

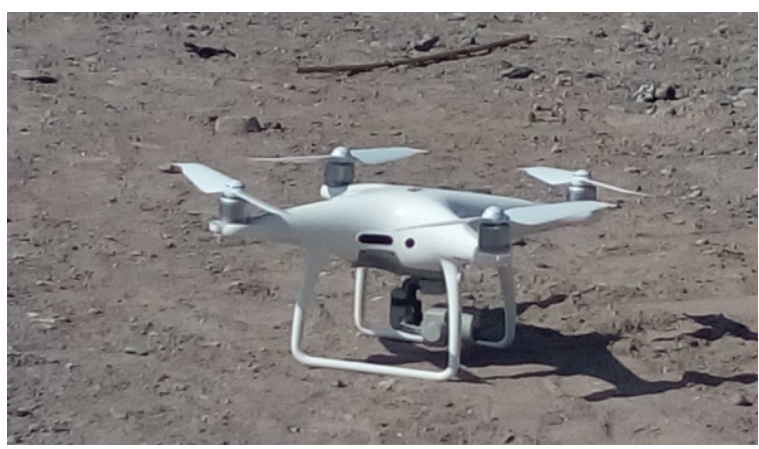

Figura 3. Drone phantom 4pro v.2.0 utilizado para el levantamiento topográfico.

\section{Ensayos geotécnicos de campo Directos}

En cuanto a los ensayos directos, se realizaron un total de 6 calicatas que fueron distribuidas en toda la zona de estudio como se observa en la Figura 4.

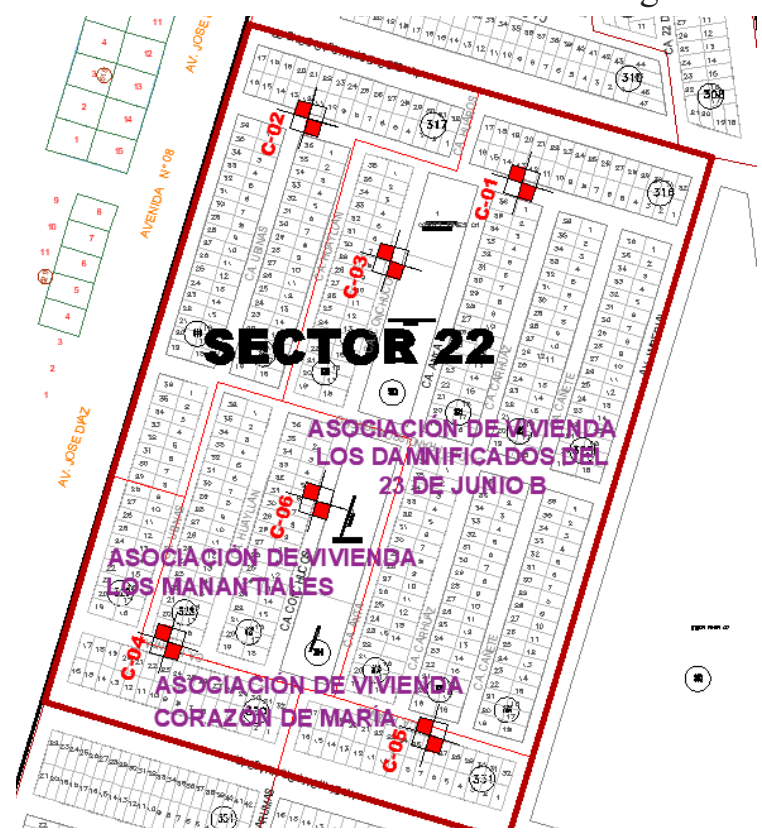

Figura 4. Ubicación de las calicatas.

Las calicatas fueron excavadas con una dimensión de $2 \mathrm{~m} * 1 \mathrm{~m}$ y con profundidades variables de 1.5 a $3 \mathrm{~m}$, cada calicata fue evaluada en campo por medio del ensayo de densidad in situ, realizado por el método del cono de arena, de acuerdo a la norma técnica peruana (NTP) 339.143 (NORMA - American Society for Testing and Materials o ASTM 1556).

De este modo, la norma técnica E.050 de Suelos y Cimentaciones nos indica las formas de obtener y transportar las muestras del terreno para la realización de los ensayos correspondientes, de las calicatas se extrajeron muestras alteradas en bolsas herméticas. 


\section{Procesamiento y análisis de datos}

El propósito principal de los ensayos geotécnicos es determinar las características de los diversos suelos presentes en la zona de estudio. E1 procesamiento y análisis de datos se ejecuta con el software SeisImager que es dispuesto por la página web Geometrics con acceso libre, en el cual se procesa la información a través de los módulos de programa según el procedimiento de (Park, Miller, Xia, \& Ivanov, 2007).
En la Tabla 1 se muestra la ubicación de las líneas geofísicas del ensayo de refracción sísmica. Este método consiste en medir el tiempo en el que la energía se propaga en forma de ondas $\mathrm{Vp}$ (ondas de compresión). El procesamiento de la información se ejecuta con el módulo de programa "PICKWIN", que determina el tiempo que tardan en propagarse las ondas sísmicas desde los puntos de tiro hasta los geófonos.

Tabla 1. Ubicación de las líneas geofísicas distribuidas en la zona de estudio. Datum: WGS84

\begin{tabular}{|c|c|c|c|c|c|c|c|c|c|}
\hline $\begin{array}{l}\text { UBICACIC } \\
\text { SÍSMICAS I }\end{array}$ & $\begin{array}{l}\text { E LAS LÍ } \\
\text { NVESTIG }\end{array}$ & 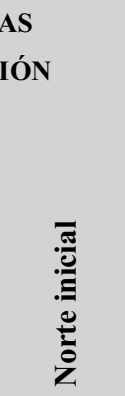 & 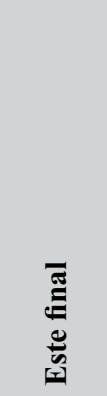 & 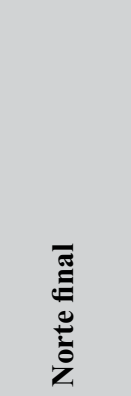 & 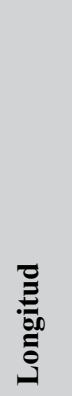 & 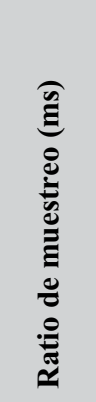 & 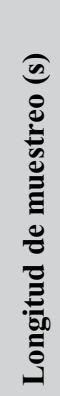 & 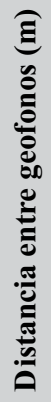 & 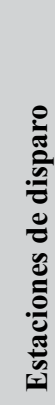 \\
\hline LS1 & 366495 & 802188 & 366566 & 8002164 & 70 & 0.125 & 0.5 & 3 & 5 \\
\hline LS2 & 366389 & 8002225 & 366461 & 8002199 & 70 & 0.125 & 0.5 & 3 & 5 \\
\hline LS3 & 366335 & 8002072 & 366407 & 8002048 & 70 & 0.125 & 0.5 & 3 & 5 \\
\hline LS4 & 366450 & 8002038 & 366523 & 8002014 & 70 & 0.125 & 0.5 & 3 & 5 \\
\hline LS5 & 366411 & 8001881 & 366483 & 8001956 & 70 & 0.125 & 0.5 & 3 & 5 \\
\hline LS6 & 366278 & 8001928 & 366351 & 8001902 & 70 & 0.125 & 0.5 & 3 & \\
\hline LS7 & 366404 & 8001984 & 366446 & 8002097 & 120 & 0.125 & 0.5 & 5 & 5 \\
\hline
\end{tabular}

En la Tabla 2 se aprecia la ubicación de los estudios MASW, este método se usa para evaluar la velocidad de onda de corte (Vs); la interpretación de los registros se realiza con el módulo de programa "SURFACE WAVE ANALYSIS", que consiste en obtener una curva de la velocidad de ondas superficiales versus la frecuencia, luego, mediante el módulo de programa "WAVE EQ" se realiza un cálculo inverso del método de inversión y a partir de la curva de dispersión se obtiene el perfil sísmico del terreno en función de Vs para cada punto de estudio.
Tabla 2. Ubicación de los estudios MASW. Datum: WGS 84

\begin{tabular}{cccccc} 
No & $\begin{array}{c}\text { ENSAYO } \\
\text { MASW }\end{array}$ & NORTE & ESTE & $\begin{array}{c}\text { UBICACIÓN } \\
\text { Ratio de } \\
\text { (ms) }\end{array}$ & $\begin{array}{c}\text { Longitud } \\
\text { de } \\
\text { muestreo } \\
\text { (s) }\end{array}$ \\
\hline 1 & MASW01 & 8002179 & 366537 & 1 & 1 \\
2 & MASW02 & 8002211 & 366423 & 1 & 1 \\
3 & MASW03 & 8002059 & 366374 & 1 & 1 \\
4 & MASW04 & 8002028 & 366490 & 1 & 1 \\
5 & MASW05 & 8001870 & 366444 & 1 & 1 \\
6 & MASW06 & 8001913 & 366319 & 1 & 1
\end{tabular}


En la Figura 5 se observa el plano con la ubicación de las 7 líneas sísmicas y 6 puntos de MASW

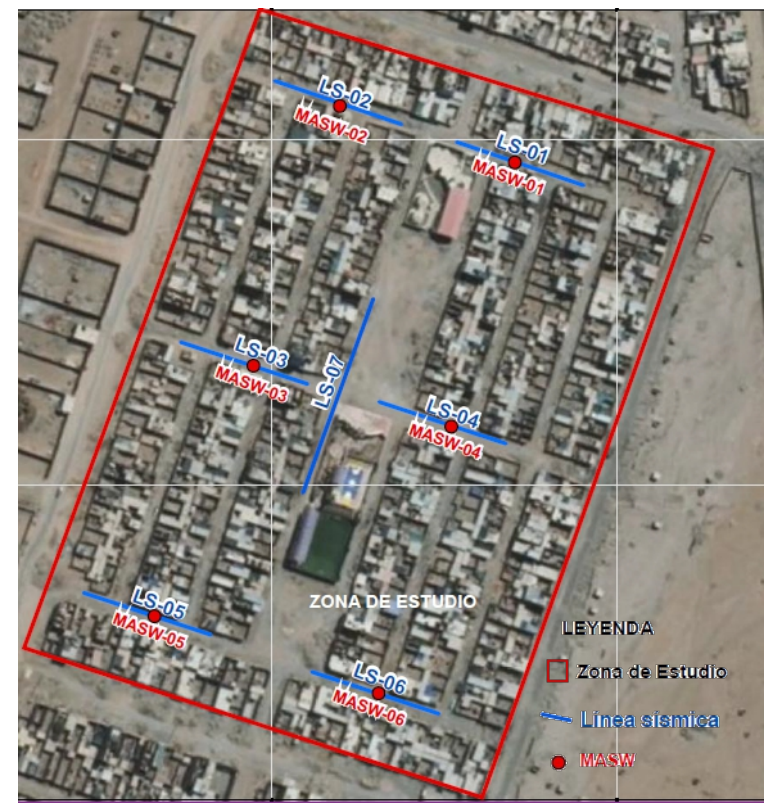

Figura 5. Plano de ubicación de las líneas sísmicas y MASW.A continuación, se señalan los ensayos de laboratorio realizados en la presente investigación.

Se realizaron los siguientes ensayos estándar: Contenido de Humedad NTP 339.127 (ASTM D2216); Análisis Granulométrico NTP 339.128
(ASTM D422); Densidad Máxima NTP 339.137 (ASTM D4253); Densidad Mínima NTP 339.138 (ASTM D4254), Densidad In situ NTP 339.143 (ASTM 1556); Ensayo de Compactación Proctor Modificado NTP 339.141 (ASTM D1557); Corrección de peso Unitario y Contenido de Humedad para partículas de sobre tamaño (ASTMD4718).

De esta forma se realizaron los siguientes ensayos especiales: Corte Directo NTP 339.171 (ASTM D3080); Compresión Triaxial no consolidado no drenado NTP 339.164 (ASTM D2850)

Asimismo se realizaron los siguientes ensayos químicos: Sulfatos NTP 400.014 (ASTM D 516); Cloruros NTP 400.014 (ASTM D 512); Contenido de Sales Solubles Totales en Suelos NTP 339.152 (BS 1377)

\section{RESULTADOS}

\section{Resultados de refracción sísmica}

En cuanto a los resultados del ensayo de refracción sísmica, se reconocieron 3 niveles de estratificación diferenciadas por sus velocidades de onda Vp. En la Tabla 3 se aprecia la descripción de los resultados obtenidos en las líneas sísmicas.

Tabla 3. Resultados obtenidos en las líneas sísmicas

\begin{tabular}{|c|c|c|c|c|c|}
\hline \multirow{3}{*}{$\begin{array}{l}\text { LÍNEA } \\
\text { SÍSMICA }\end{array}$} & \multicolumn{4}{|c|}{ POTENCIA } & \multirow[b]{3}{*}{ Material Coluvial. } \\
\hline & \multirow{2}{*}{$\begin{array}{c}\text { CAPA } \\
\text { A }\end{array}$} & \multicolumn{3}{|c|}{$\begin{array}{l}\text { PROMEDIO Vp (m/s) } \\
\quad(\mathrm{m})\end{array}$} & \\
\hline & & 2 & 300 & MATERIALES SUELTOS & \\
\hline \multirow[t]{3}{*}{-01 y LS-02 } & B & 5 & 1000 & RIPADO FACIL & Material Coluvial con saturación. \\
\hline & $\mathrm{C}$ & 18 & 2000 & RIPADO DURO - VOLADURA & Material-fluvial \\
\hline & A & $1-1.5$ & 300 & MATERIALES SUELTOS & Material Coluvial. \\
\hline \multirow[t]{3}{*}{-03 y LS-04 } & B & 4.5 & 1000 & RIPADO FACIL & Material Coluvial con saturación. \\
\hline & $\mathrm{C}$ & 14 & 2000 & RIPADO DURO - VOLADURA & Material-fluvial \\
\hline & A & $1-1.5$ & 300 & MATERIALES SUELTOS & Material Coluvial. \\
\hline \multirow[t]{3}{*}{-05 y LS-06 } & B & 4.5 & 1000 & RIPADO FACIL & Material Coluvial con saturación. \\
\hline & $\mathrm{C}$ & 14 & 2000 & RIPADO DURO - VOLADURA & Material-fluvial Coluvial \\
\hline & A & 1.5 & 300 & MATERIALES SUELTOS & Material Coluvial. \\
\hline \multirow[t]{2}{*}{ LS-07 } & B & 5 & 1000 & RIPADO FACIL & Material Coluvial con saturación. \\
\hline & $\mathrm{C}$ & 10 & 2000 & RIPADO DURO - VOLADURA & Material-fluvial \\
\hline
\end{tabular}


De acuerdo a los resultados obtenidos del ensayo de refracción sísmica, se distinguieron 3 niveles de estratificación: la capa A, B y C. Estas se determinan a continuación:

Estrato A: con velocidades de onda Vp 300 m/s; correspondiente a materiales sueltos no compactos siendo correlacionados por material suelto. Tiene una potencia menor a $5 \mathrm{~m}$.

Estrato B: con velocidades de onda Vp $1000 \mathrm{~m} / \mathrm{s}$, correspondiente a materiales semicompactos representados por conglomerados. Tiene una potencia menor a $8 \mathrm{~m}$.

Estrato C: con velocidades de onda Vp $2000 \mathrm{~m} / \mathrm{s}$, correspondientes a materiales semicompactos con mayor densidad (aumento de la Vp), representados por conglomerados. Tiene una potencia menor a $10 \mathrm{~m}$. De este modo, la velocidad sísmica de propagación de las ondas de compresión (Ondas Vp) es el parámetro más representativo para la ripabilidad del terreno. De esta manera, Caterpillar publica tablas de ripabilidad para los distintos modelos de tractores a través de la velocidad de onda Vp (Rodríguez, 2001).

Por otra parte, se realizó el procesamiento de datos. En la Figura 5 se observa el perfil de la línea sísmica LS-01.

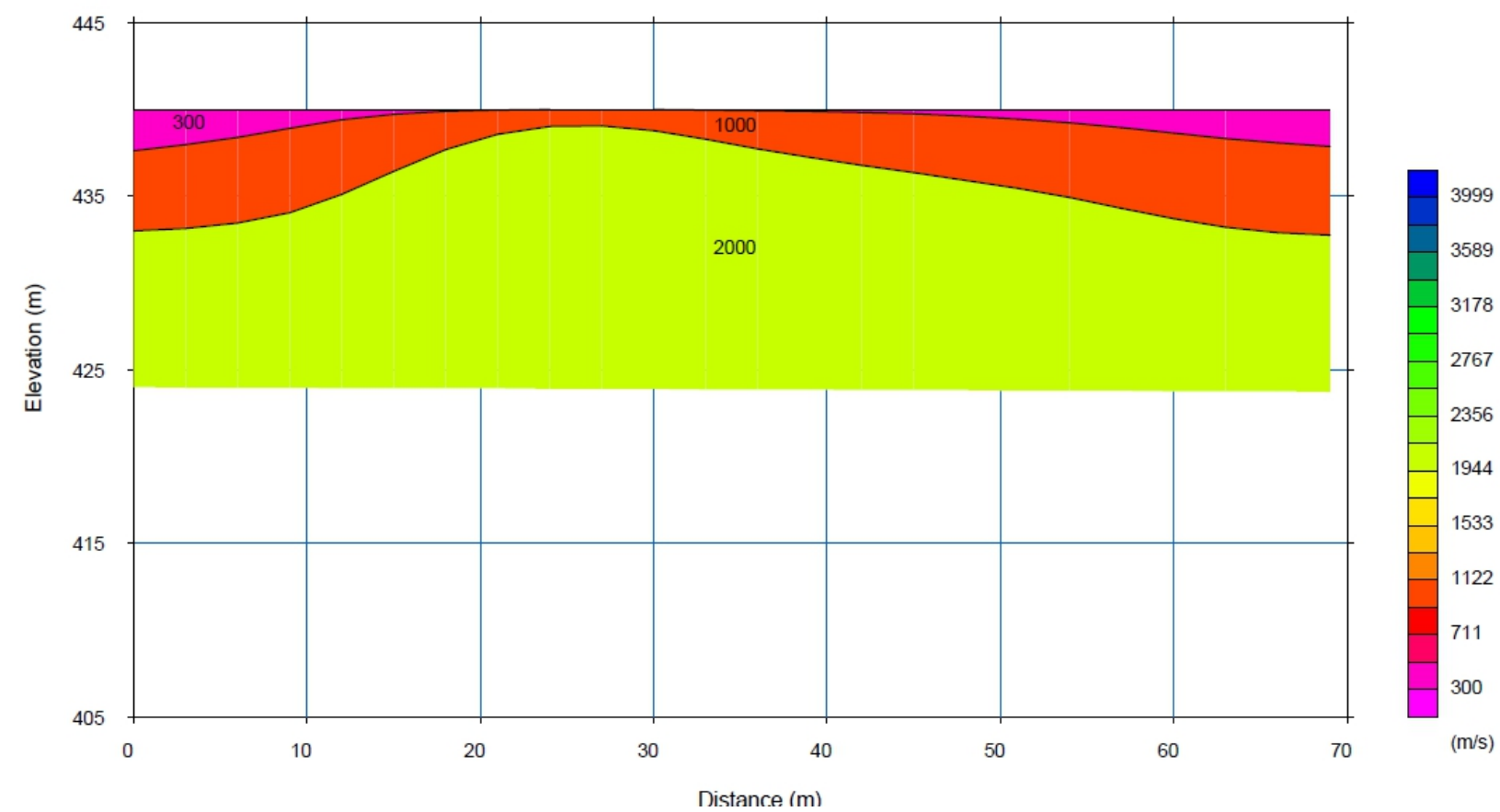

Figura 6. Perfil estratigráfico LS-02.

\section{Resultados de MASW}

De igual forma, del ensayo de MASW se determinó el perfil unidimensional de velocidades de onda S (Vs) hasta una profundidad de $30 \mathrm{~m}$. De este modo, se clasificó el tipo de suelo conforme a la NTE E.030 y se calculó el periodo fundamental de vibración del suelo Ts, tener en cuenta que este valor es referencial. Para este estudio se usó la fórmula según el código International Building Code (2012):

Donde:

$$
T s=\frac{4 H}{V s_{30}}
$$

$\mathrm{H}=\mathrm{La}$ potencia del estrato para nosotros $30 \mathrm{~m}$; $\mathrm{Vs}_{30}=$ El valor de la onda de corte Vs promedio.
En el Tabla 4 se aprecia la clasificación Vs30, con respecto a la Norma Sismorresistente (NTE) E.030 y periodo de vibración.

Tabla 4. Clasificación Sismorresistente E.030 y periodo de vibración Ts

\begin{tabular}{|c|c|c|c|c|c|c|}
\hline 离 & $\begin{array}{l}5 \\
\dot{1} \\
3 \\
3 \\
\sum\end{array}$ & 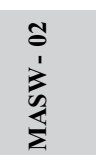 & $\begin{array}{l}0 \\
0 \\
1 \\
\vdots \\
\sum \\
\sum\end{array}$ & $\begin{array}{l} \pm \\
0 \\
1 \\
\vdots \\
\sum \\
\sum\end{array}$ & $\begin{array}{l}10 \\
0 \\
1 \\
3 \\
\frac{1}{2}\end{array}$ & $\begin{array}{l}0 \\
\vdots \\
1 \\
3 \\
5 \\
\sum\end{array}$ \\
\hline $\begin{array}{l}\text { Tipo } \\
\text { Suelo }\end{array}$ & S1 & S1 & S1 & S1 & S1 & S1 \\
\hline $\begin{array}{l}\text { Vs30 } \\
(\mathrm{m} / \mathrm{s})\end{array}$ & 658.1 & 677.4 & 639.8 & 653.9 & 645.6 & 642.1 \\
\hline Ts & $\begin{array}{l}0.18 \\
\text { ROCA }\end{array}$ & $\begin{array}{c}0.18 \\
\text { - SUEL }\end{array}$ & 0.19 & 0.18 & $\begin{array}{c}0.19 \\
\text { RO } \\
\text { SUF } \\
\text { RIG }\end{array}$ & $\begin{array}{l}0.19 \\
\text { LAO } \\
\text { LOS } \\
\text { DOS }\end{array}$ \\
\hline
\end{tabular}


De acuerdo a la Norma Técnica Peruana Sismorresistente (NTE) E.030 el tipo de suelo es S1 correspondiente a suelos muy rígidos, con una Vs30 variante desde $639.8 \mathrm{~m} / \mathrm{s}$ a $677.4 \mathrm{~m} / \mathrm{s}$ y un periodo de

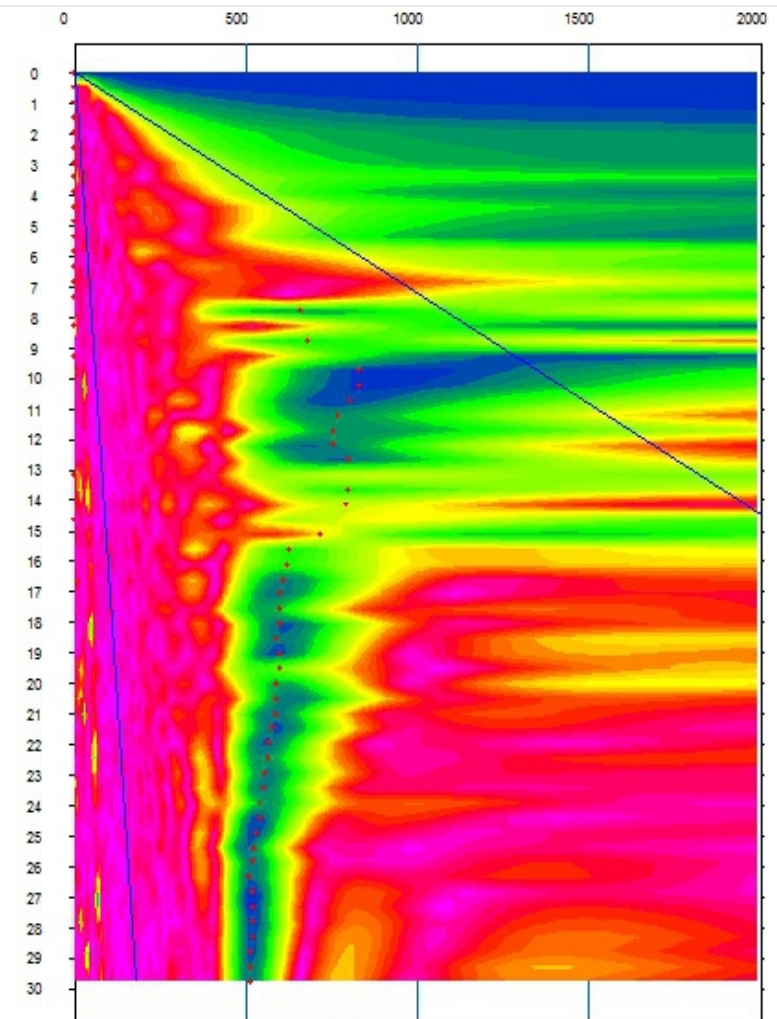

Figura 7. Procesamiento del registro sísmico MASW-02.

\section{Resultados de los ensayos de laboratorio}

Posteriormente, los ensayos de laboratorio que se realizaron a las muestras de la zona de estudio. En la Tabla 5 se aprecia el resumen de los resultados de los ensayos estándar.

Tabla 5. Resultados de los ensayos de laboratorio

\begin{tabular}{|c|c|c|c|c|c|c|c|c|}
\hline 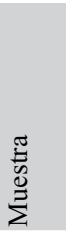 & 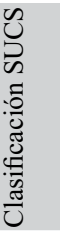 & 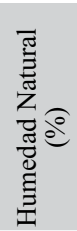 & 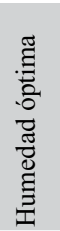 & 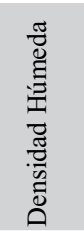 & 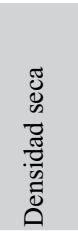 & 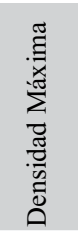 & 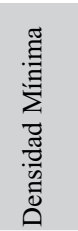 & 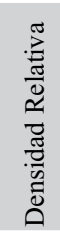 \\
\hline $\mathrm{C}-01$ & GP & 1.58 & 6.1 & 2.197 & 2.163 & 2.209 & 2.112 & 53.4 \\
\hline $\mathrm{C}-02$ & GP & 1.72 & 8.7 & 2.14 & 2.104 & 0.153 & 2.048 & 54.3 \\
\hline $\mathrm{C}-03$ & GP & 1.79 & 9.4 & 2.159 & 2.121 & 2.178 & 2.057 & 54 \\
\hline $\mathrm{C}-04$ & GP & 1.56 & 9.7 & 2.105 & 2.073 & 2.151 & 1.991 & 53.2 \\
\hline $\mathrm{G}-05$ & $\mathrm{P} P$ & 1.43 & 7.8 & 2.080 & 2.050 & 2.113 & 1.986 & 52.1 \\
\hline$=06$ & $P$ & 1.69 & 9.5 & 2.177 & 2.141 & 2.183 & 2.096 & 52.9 \\
\hline
\end{tabular}

Los ensayos de límite líquido y límite plástico no se pudieron completar, ya que el suelo no presenta las características necesarias. vibración del suelo (Ts) que va desde 0.18 a 0.19 , teniendo un promedio de Ts de 0.18 .

En la Figura 6, se muestra el análisis de la dispersión de ondas para ser invertidos en un perfil Vs de 1D.

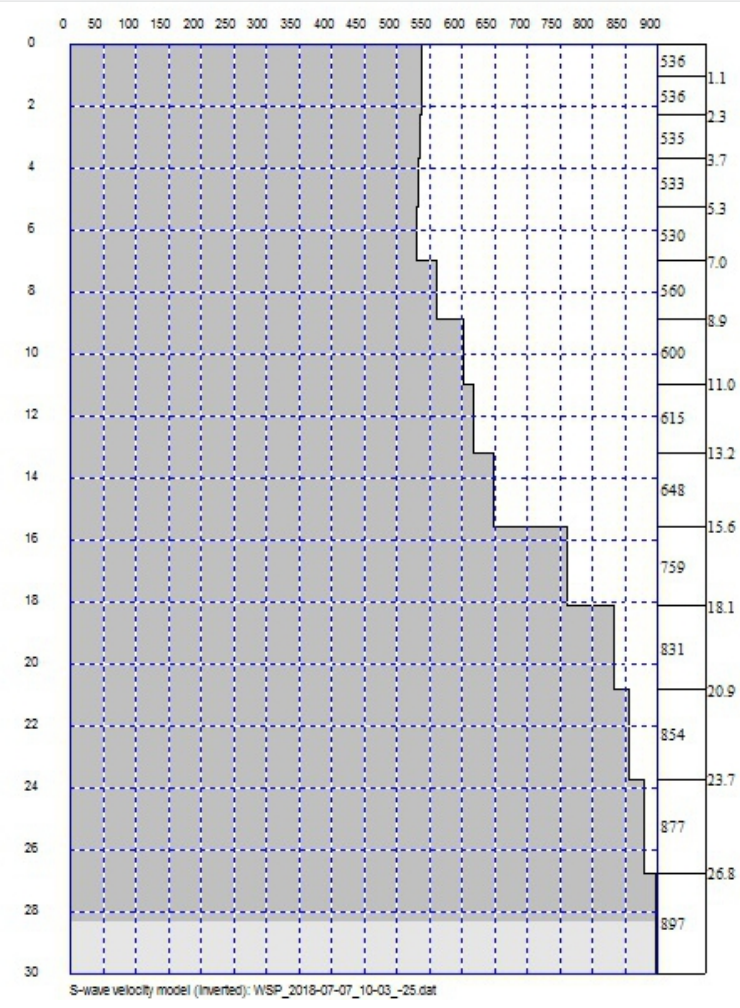

\section{Resultados de los ensayos especiales}

Los ensayos especiales, de corte directo y triaxial, fueron realizados sobre muestras alteradas, las cuales se extrajeron y se transportaron en bolsas impermeables, estos ensayos determinan el ángulo de fricción y el coeficiente de cohesión. En la Tabla 6 se muestra los resultados de los ensayos de corte directo y triaxial.

Tabla 6. Resultados de los ensayos de Corte Directo y Triaxial

\begin{tabular}{ccccc} 
Muestra & $\begin{array}{c}\text { Ensayo de Corte } \\
\text { Directo } \\
\text { Ángulo } \\
\text { de } \\
\text { fricción } \\
\left({ }^{\circ}\right)\end{array}$ & $\begin{array}{c}\text { Coeficiente } \\
\text { de } \\
\text { Cohesión } \\
\left(\mathrm{kg} / \mathrm{cm}^{2}\right)\end{array}$ & $\begin{array}{c}\text { Ensayo de } \\
\text { Compresión Triaxial } \\
\text { Ángulo } \\
\text { fricción } \\
\left({ }^{\circ}\right)\end{array}$ & $\begin{array}{c}\text { Coeficiente } \\
\text { de } \\
\text { Cohesión } \\
\left(\mathrm{kg} / \mathrm{cm}^{2}\right)\end{array}$ \\
\hline C-01 & 32.47 & 0.082 & - & - \\
C-02 & 35.66 & 0.114 & 32.47 & 0.00 \\
C-03 & 35.53 & 0.114 & 31.78 & 0.06 \\
C-04 & 37.11 & 0.130 & - & - \\
C-05 & 36.95 & 0.134 & 33.76 & 0.03 \\
C-06 & 37.80 & 0.124 & - & - \\
\hline
\end{tabular}




\section{Resultados de los ensayos químicos}

Para determinar el grado de agresividad química del suelo frente a una cimentación, se analizaron químicamente las muestras del suelo mediante los ensayos de cloruros, sulfatos y contenido de sales solubles. En la Tabla 7 se muestra los resultados de los ensayos químicos.

Tabla 7. Resultados de los ensayos químicos

\begin{tabular}{|c|c|c|c|c|c|c|}
\hline 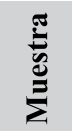 & $\frac{\mathscr{c}}{\stackrel{0}{0}}$ & $\frac{0}{\frac{0}{\pi}}$ & 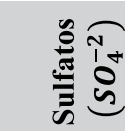 & & $\stackrel{a}{\grave{z}}$ & \\
\hline C- 01 & $0.141 \%$ & $\begin{array}{l}1408 \\
\text { ppm }\end{array}$ & $0.058 \%$ & $\begin{array}{c}577.3 \\
\mathrm{ppm}\end{array}$ & $0.072 \%$ & $\begin{array}{l}718.1 \\
\mathrm{ppm}\end{array}$ \\
\hline C- 02 & $0.669 \%$ & $\begin{array}{l}6688 \\
\mathrm{ppm}\end{array}$ & $0.274 \%$ & $\begin{array}{c}2742.1 \\
\mathrm{ppm}\end{array}$ & $0.341 \%$ & $\begin{array}{c}3410.9 \\
\text { ppm }\end{array}$ \\
\hline $\mathrm{C}-03$ & $0.046 \%$ & $\begin{array}{c}457.6 \\
\mathrm{ppm}\end{array}$ & $0.019 \%$ & $\begin{array}{c}187.6 \\
\mathrm{ppm}\end{array}$ & $0.023 \%$ & $\begin{array}{r}233.1 \\
\mathrm{ppm}\end{array}$ \\
\hline C-04 & $0.385 \%$ & $\begin{array}{c}3846.4 \\
\mathrm{ppm}\end{array}$ & $0.158 \%$ & $\begin{array}{l}1577 \\
\mathrm{ppm}\end{array}$ & $0.196 \%$ & $\begin{array}{c}1961.7 \\
\mathrm{ppm}\end{array}$ \\
\hline C- 05 & $0.121 \%$ & $\begin{array}{c}1213.4 \\
\mathrm{ppm}\end{array}$ & $0.050 \%$ & $\begin{array}{c}497.5 \\
\mathrm{ppm}\end{array}$ & $0.062 \%$ & $\begin{array}{c}618.8 \\
\mathrm{ppm}\end{array}$ \\
\hline C-06 & $0.093 \%$ & $\begin{array}{c}928.6 \\
\text { ppm }\end{array}$ & $0.038 \%$ & $\begin{array}{c}380.7 \\
\mathrm{ppm}\end{array}$ & $0.047 \%$ & $\begin{array}{r}473.6 \\
\mathrm{ppm}\end{array}$ \\
\hline
\end{tabular}

\section{Cálculo de la capacidad de carga}

El fin de este estudio se basó en la información recopilada de los ensayos de laboratorio del suelo. La capacidad portante para las 6 calicatas se calculó a través de la Teoría de capacidad de carga de Terzaghi y Peck (1967), con los factores de carga propuestos por Vesic (1973) y la expresión de Meyerhof(1963).

De esta forma, se obtuvo:

En la calicata 1, la capacidad portante para una cimentación corrida de $0.5 \mathrm{~m}$ de ancho cimentada a una profundidad de 0.8 a $1.5 \mathrm{~m}$ varía de $0.65 \mathrm{a}$ $1.08 \mathrm{~kg} / \mathrm{cm}^{2}$, para una cimentación cuadrada de 1 $\mathrm{m}$ de ancho cimentada a una profundidad de $1 \mathrm{a}$ $2.5 \mathrm{~m}$ varía de 1.07 a $2.40 \mathrm{~kg} / \mathrm{cm}^{2}$, para una cimentación rectangular de $1.2 \mathrm{~m}$ de ancho y 1.4 de largo cimentada a una profundidad de 1 a $2.5 \mathrm{~m}$ varía de 1.08 a $2.36 \mathrm{~kg} / \mathrm{cm}^{2}$.

En la calicata 2, la capacidad portante para una cimentación corrida de $0.5 \mathrm{~m}$ de ancho cimentada a una profundidad de 0.8 a $1.5 \mathrm{~m}$ varía de $0.84 \mathrm{a}$ $1.40 \mathrm{~kg} / \mathrm{cm}^{2}$, para una cimentación cuadrada de 1 $\mathrm{m}$ de ancho cimentada a una profundidad de $1 \mathrm{a}$
$2.5 \mathrm{~m}$ varía de 1.42 a $3.18 \mathrm{~kg} / \mathrm{cm}^{2}$, para una cimentación rectangular de $1.2 \mathrm{~m}$ de ancho y 1.4 de largo cimentada a una profundidad de 1 a $2.5 \mathrm{~m}$ varía de 1.44 a $3.12 \mathrm{~kg} / \mathrm{cm}^{2}$.

En la calicata 3, la capacidad portante para una cimentación corrida de $0.5 \mathrm{~m}$ de ancho cimentada a una profundidad de 0.8 a $1.5 \mathrm{~m}$ varía de 0.84 a $1.39 \mathrm{~kg} / \mathrm{cm}^{2}$, para una cimentación cuadrada de 1 $\mathrm{m}$ de ancho cimentada a una profundidad de 1 a $2.5 \mathrm{~m}$ varía de 1.41 a $3.17 \mathrm{~kg} / \mathrm{cm}^{2}$, para una cimentación rectangular de $1.2 \mathrm{~m}$ de ancho y 1.4 de largo cimentada a una profundidad de 1 a $2 \mathrm{~m}$ varía de 1.44 a $2.55 \mathrm{~kg} / \mathrm{cm}^{2}$.

En la calicata 4, la capacidad portante para una cimentación corrida de $0.5 \mathrm{~m}$ de ancho cimentada a una profundidad de 0.8 a $1.5 \mathrm{~m}$ varía de 0.95 a $1.58 \mathrm{~kg} / \mathrm{cm}^{2}$, para una cimentación cuadrada de 1 $\mathrm{m}$ de ancho cimentada a una profundidad de 1 a 2 $\mathrm{m}$ varía de 1.63 a $2.97 \mathrm{~kg} / \mathrm{cm}^{2}$, para una cimentación rectangular de $1.2 \mathrm{~m}$ de ancho y 1.4 de largo cimentada a una profundidad de 1 a $1.5 \mathrm{~m}$ varía de 1.65 a $2.29 \mathrm{~kg} / \mathrm{cm}^{2}$.

En la calicata 5, la capacidad portante para una cimentación corrida de $0.5 \mathrm{~m}$ de ancho cimentada a una profundidad de 0.8 a $1.5 \mathrm{~m}$ varía de 0.93 a $1.54 \mathrm{~kg} / \mathrm{cm}^{2}$, para una cimentación cuadrada de 1 $\mathrm{m}$ de ancho cimentada a una profundidad de 1 a 2 $\mathrm{m}$ varía de 1.58 a $2.88 \mathrm{~kg} / \mathrm{cm}^{2}$, para una cimentación rectangular de $1.2 \mathrm{~m}$ de ancho y 1.4 de largo cimentada a una profundidad de 1 a $1.5 \mathrm{~m}$ varía de $1.61 \mathrm{a} 2.23 \mathrm{~kg} / \mathrm{cm}^{2}$.

En la calicata 6, la capacidad portante para una cimentación corrida de $0.5 \mathrm{~m}$ de ancho cimentada a una profundidad de 0.8 a $1.5 \mathrm{~m}$ varía de 1.06 a $1.74 \mathrm{~kg} / \mathrm{cm}^{2}$, para una cimentación cuadrada de 1 $\mathrm{m}$ de ancho cimentada a una profundidad de $1 \mathrm{a}$ $1.5 \mathrm{~m}$ varía de 1.81 a $2.55 \mathrm{~kg} / \mathrm{cm}^{2}$, para una cimentación rectangular de $1.2 \mathrm{~m}$ de ancho y 1.4 de largo cimentada a una profundidad de 1 a $1.5 \mathrm{~m}$ varía de 1.84 a $2.54 \mathrm{~kg} / \mathrm{cm}^{2}$.

Asimismo, se halló la capacidad portante para los ángulos de fricción obtenidos por el triaxial, siendo hallados con la Teoría de Meyerhof para la falla general y localizada.

En la calicata 2, se obtuvo una capacidad portante por falla local para una cimentación cuadrada de 1 $\mathrm{m}$ de ancho cimentada a una profundidad de 1.5 $\mathrm{m}$, siendo para el ángulo obtenido por el triaxial $1.63 \mathrm{~kg} / \mathrm{cm}^{2}$ y por el corte directo $2.25 \mathrm{~kg} / \mathrm{cm}^{2}$. 
En la calicata 3, se obtuvo una capacidad portante por falla local para una cimentación cuadrada de 1 $\mathrm{m}$ de ancho cimentada a una profundidad de $1.5 \mathrm{~m}$, siendo para el ángulo obtenido por el triaxial 1.53 $\mathrm{kg} / \mathrm{cm}^{2}$ y por el corte directo $2.24 \mathrm{~kg} / \mathrm{cm}^{2}$.

En la calicata 5, se obtuvo una capacidad portante por falla local para una cimentación cuadrada de 1 $\mathrm{m}$ de ancho cimentada a una profundidad de $1.5 \mathrm{~m}$, siendo para el ángulo obtenido por el triaxial 1.80 $\mathrm{kg} / \mathrm{cm}^{2}$ y por el corte directo $2.52 \mathrm{~kg} / \mathrm{cm}^{2}$.

Para las capacidades portantes mencionadas anteriormente los asentamientos calculados, los cuales se encuentran por debajo del valor límite recomendado de $2.54 \mathrm{~cm}$ para cada una de las calicatas.

\section{DISCUSIÓN}

Los resultados de la presente investigación fueron obtenidos a través de los ensayos de laboratorio de mecánica de suelos, y los métodos geofísicos de refracción sísmica y MASW, quedando registros de este estudio por medio de fotografías, además de resultados de los ensayos de mecánicas suelo y geofísicos. Los resultados obtenidos del área de estudio nos otorgan los datos para poder dar un diseño de cimentación adecuado para esta zona.

De este modo, los resultados obtenidos solo podrán ser aplicados a esta zona, pero la metodología del estudio puede ser aplicado a otras investigaciones, debido que los ensayos geofísicos y ensayos de laboratorio pueden encontrar las verdaderas propiedades del suelo (tanto dinámicas, físicas, químicas y mecánicas). Dentro de las limitaciones que existieron en el desarrollo de esta investigación, se puede citar a tres, considerando las más importantes: Transporte de material, remoldeo del material y tiempo de ejecución de los ensayos de laboratorio.

La primera corresponde a que los ensayos básicos y especiales de laboratorio fueron analizados en la Universidad Nacional del Altiplano, situada en la ciudad de Puno, siendo transportado casi $300 \mathrm{~kg}$ de material de las 6 calicatas, realizadas en la zona de estudio ubicada en la ciudad de Tacna.

La segunda se ocasionó debido a que el suelo es conformado por grava mal graduada, de este modo la muestra no pudo ser extraída de forma inalterada, siendo remodelada en el laboratorio para la realización de los ensayos especiales.

La tercera, debido a la cantidad de material a ser analizado y cambio de personal en el laboratorio, lo que ocasionó un retraso en la entrega de resultados.

Dentro de los resultados obtenidos, es importante resaltar que la mayoría de ellos son similares a investigaciones anteriores, desarrolladas en el distrito de Gregorio Albarracín Lanchipa. Comparando los resultados con el estudio realizado por INDECI (2004), la similitud está basada en la capacidad portante que se espera que sea mayor a $3 \mathrm{~kg} / \mathrm{cm}^{2}$. Se halló en nuestro estudio una capacidad portante para la profundidad $1.2 \mathrm{y}$ una zapata cuadrada de $1 \mathrm{~m} \times 1 \mathrm{~m}$ de $4.17 \mathrm{~kg} / \mathrm{cm}^{2}$ para ser comparada con dicho estudio.

De esta manera, se obtuvo una diferencia de resultados en la exposición a los sulfatos. Según INDECI (2004) propuso un valor máximo de $0.0498 \%$, siendo éste una exposición despreciable, lo cual no coincide con los valores obtenidos en la calicata 2 y 4 , donde se muestra una exposición severa de acuerdo a la norma NTE E- 0.60 , siendo el valor más alto de $0.272421 \%$, el cual es casi 6 veces mayor que el dato otorgado por INDECI (2004).

Según la clasificación del suelo que se realizó en la zona de estudio por INDECI (2004) lo clasifico como un GW (grava bien graduada), mientras que con la presente investigación se obtuvo una clasificación de GP (grava pobremente graduada).

En los resultados de agresividad por sales, según Vargas (2017), la similitud se basa en la agresividad por sales, por ello, da recomendaciones para la protección de las viviendas ante este problema. En nuestro estudio se halla una agresividad de sulfatos muy alta de $0.272421 \%$. Comparando los resultados obtenidos de capacidad portante por el ensayo de MASW y ensayos de corte directo (Tezcan, Ozdemir, Keceli \& Erkal, 2007).

Para la calicata 1, por el ensayo de MASW se obtiene $1.19 \mathrm{~kg} / \mathrm{cm}^{2}$ y por el método tradicional se obtiene $1.24 \mathrm{~kg} / \mathrm{cm}^{2}$.

Para la calicata 2, por el ensayo de MASW se obtiene $1.29 \mathrm{~kg} / \mathrm{cm}^{2}$ y por el método tradicional se obtiene $1.67 \mathrm{~kg} / \mathrm{cm}^{2}$.

Para la calicata 3, por el ensayo de MASW se obtiene $1.26 \mathrm{~kg} / \mathrm{cm}^{2}$ y por el método tradicional se obtiene $1.66 \mathrm{~kg} / \mathrm{cm}^{2}$. 
Para la calicata 4, por el ensayo de MASW se obtiene $1.12 \mathrm{~kg} / \mathrm{cm}^{2}$ y por el método tradicional se obtiene $1.92 \mathrm{~kg} / \mathrm{cm}^{2}$.

Para la calicata 5, por el ensayo de MASW se obtiene $1.11 \mathrm{~kg} / \mathrm{cm}^{2}$ y por el método tradicional se obtiene $1.86 \mathrm{~kg} / \mathrm{cm}^{2}$.

Para la calicata 6, por el ensayo de MASW se obtiene $1.26 \mathrm{~kg} / \mathrm{cm}^{2}$ y por el método tradicional se obtiene $2.13 \mathrm{~kg} / \mathrm{cm}^{2}$.

Llegando a la conclusión que, el método geofísico es muy conservado y el método tradicional da resultados más reales. Teniendo coherencia en la conclusión con el estudio realizado en Ecuador por Aguay y Dranichnikov (2012).

De acuerdo a los resultados obtenidos de este estudio se conoce que la amplificación sísmica que tendrá el suelo es de un S1, según la NTE E.030 (2018).

\section{CONCLUSIÓN}

Los métodos geofísicos nos ayudan a conocer las propiedades dinámicas del suelo, la cual nos permite realizar un buen diseño de cimentación. De acuerdo a los resultados obtenidos del método geofísico de Refracción sísmica se puede afirmar que el área estudiada posee 3 diferentes capas de estratigrafía correspondiente a material suelto no compacto, material semicompacto representado por conglomerado y materiales semicompactos con mayor densidad. Eel terreno posee velocidades de onda $\mathrm{Vp}$ variante desde 300 a 2 $000 \mathrm{~m} / \mathrm{s}$. De la misma manera, con los resultados obtenidos del método de MASW se conoce que el tipo de suelo del área estudiada es un $\mathrm{S}_{1}$ correspondiente a suelos muy rígidos; con un $\mathrm{V}_{\mathrm{S30}}$ (promedio) de $652.86 \mathrm{~m} / \mathrm{s}$ y un $\mathrm{T}_{\mathrm{s}}$ (promedio) de 0.18 .

De acuerdo a los resultados obtenidos de la realización de los ensayos de laboratorio se confirmó que el suelo del área estudiada se clasifica como grava pobremente graduada (GP), el cual no presenta límite líquido ni límite plástico, con una densidad In situ que varía de 2.050 a 2.163 De esta manera se obtuvo el ángulo de fricción que varía de $32.47^{\circ}$ a $37.8^{\circ}$, de igual forma se halló tres ángulos de fricción mediante el Ensayo de Compresión Triaxial de las calicatas 2, 3 y 5 , siendo correspondientemente $32.47^{\circ}$, $31.78^{\circ}$ y $33.76^{\circ}$. Se reconoció también que presenta niveles altos de sulfatos que varían de 0.01876 a $0.27421 \mathrm{v} \%$.

Mediante los métodos geofísicos se determinaron las propiedades dinámicas del área evaluada conociendo así el módulo de Poison (v) que varía de 0.41 a 0.46 , el módulo de Young (E) que varía de 1364.28 a $4069.13 \mathrm{MPa}$, un módulo de Rigidez(G) que varía de 466.85 a $1438.80 \mathrm{MPa}$ y un módulo de BULK (K)que varía de 5853.71 a $7892.67 \mathrm{Mpa}$.

Mediante la realización de los ensayos se concluyó que es un buen suelo, apto para construcciones y que no fallaría rápidamente, siendo su capacidad portante para una cimentación cuadrada de $1 \mathrm{~m}$. cimentada a una profundidad de $1.5 \mathrm{~m}$. que varía de 1.51 a 2.55 $\mathrm{kg} / \mathrm{cm}^{2}$

\section{REFERENCIAS BIBLIOGRÁFICAS}

ASTM D 1556 (1996). Standard Test Method for density and unit weight of soil in place by the sand-cone method. USA: American Society for Testing and Materials.

Aguay, H. y Dranichnikov, T. (2012). Cálculo de capacidad portante basado en geofisica y método convencional: El caso del puente sobre el río Maicito. Ecuador: Universidad Politécnica Salesiana.

Hayashi, K. (2008). Development of the Surfacewave Methods and Its Application to Site Investigations (Thesis or Dissertation). Kyoto University, Japón.

Huanacuni, D. y Llanqui, G. (2014). Caracterización dinámica de los suelos de la ciudad de Tacna (tesis de pregrado). Universidad Privada de Tacna, Tacna, Perú.

IBC. (2012). International Building Code.USA: International Code Council.

INDECI. (2004). Mapa de peligros de la ciudad de Tacna: distritos de Tacna, Gregorio Albarracín, Pocollay y Complemento Alto de la Alianza y Ciudad Nueva. Volumen I-Memoria, Tacna.

Meyerhof, G. (1963). Some Recent Research on the Bearing Capacity of Foundations The ultimate bearing capacity of foundations. Canadian Geotechnical Journal, 1, 16-26.

NTP 339.143 (1999). Método de ensayo estándar 
para la densidad y peso unitario del suelo in-situ mediante el método del cono de arena. Lima, Perú:Comisión de Reglamentos Técnicos y Comerciales

NTE E.030 (2018). Diseño sismorresistente. Lima, Perú: Diario Oficial El Peruano.

NTE E.050 (2018). Suelos y Cimentaciones. Lima, Perú: Diario Oficial El Peruano.

Park, C., Miller, R., \& Xia, J. (1999). Multichannel analysis of surface waves (MASW): Geophysics, 64(3), 800-808.

Park, C., Miller, R., Xia, J., \& Ivanov, J. (2007). Multichannel analysis of surface waves (MASW)- active and passive methods: $T h e \quad L e a d i n g \quad E d g e$. Recuperado de http://www.masw.com /files/PAR-07-A.PDF

Rivera, M., Piedra, R., y Paripanca, Y. (2016). Ensayos Geofísicos de Refracción Sísmica y de Medición de Ondas de Corte (MASW y MAM) para usos de cimentación en obras de edificaciones. Civilizate-CIV. 8, 50-55 Recuperado de http://revistas.pucp.edu.pe/index.ph $\mathrm{p} /$ civilizate/article/view/18630

Rodríguez, M. (2001). Geofisica aplicada a la obra civil: Método geoeléctrico y sísmica de refracción. Recuperado de http://www.geoconsult.es

Rosales, C. (2001). Sobre el comportamiento sísmico de los depósitos de suelos del área de Cañaveralejo, Cali, Colombia (tesis de pregrado). Universidad del Valle, Santiago de Cali, Colombia.

Terzaghi, K. \& Peck, R. (1967). Soil Mechanics in Engineering Practice (Second ed.). New York: John Wiley \& Sons, Inc.

Tezcan, S., Ozdemir, Z., Keceli, A. \& Erkal, A. (octubre, 2007). A Rapid Technique to Determine Allowable Bearing Ressure.International Earthquake symposium Kocaeli. Simposio llevado a cabo en el Kocaeli Metropolitan Municipality Convention, Turquia.

Vara, A. (2017). Desde la idea a la sustentación :7 pasos para una tesis exitosa. Lima: Universidad de San Martin de Porres.

Vargas, G. (2017). Diagnóstico, prevención y reparación en viviendas de albañilería con problemas de humedad en el distrito Gregorio Albarracín Lanchipaprovincia Tacna-departamento Tacna (tesis de pregrado). Universidad Privada de Tacna, Tacna, Perú.

Vesic, A. (1973). Analysis of ultimate loads of shallow foundations. Journal of the Soil Mechanics and Foundations Division, 99, 45-73. Recuperado de https://cedb.asce.org/CEDBsearch/rec ord.jsp? dockey $=0020165$ 